University of Nebraska - Lincoln

DigitalCommons@University of Nebraska - Lincoln

U.S. National Park Service Publications and

Papers

National Park Service

$2-25-2020$

\title{
Estimation of available epinephrine dose in expired and discolored autoinjectors via quantitative smartphone imaging
}

Amirus Saleheen

Bill M. Campbell

Rebecca A. Prosser

Christopher A. Baker

Follow this and additional works at: https://digitalcommons.unl.edu/natlpark

Part of the Environmental Education Commons, Environmental Policy Commons, Environmental

Studies Commons, Fire Science and Firefighting Commons, Leisure Studies Commons, Natural Resource Economics Commons, Natural Resources Management and Policy Commons, Nature and Society Relations Commons, Other Environmental Sciences Commons, Physical and Environmental Geography Commons, Public Administration Commons, and the Recreation, Parks and Tourism Administration Commons

This Article is brought to you for free and open access by the National Park Service at DigitalCommons@University of Nebraska - Lincoln. It has been accepted for inclusion in U.S. National Park Service Publications and Papers by an authorized administrator of DigitalCommons@University of Nebraska - Lincoln. 


\title{
Estimation of available epinephrine dose in expired and discolored autoinjectors via quantitative smartphone imaging
}

\author{
Amirus Saleheen ${ }^{1} \cdot$ Bill M. Campbell ${ }^{2,3} \cdot$ Rebecca A. Prosser ${ }^{3,4} \cdot$ Christopher A. Baker $^{1,3}$ (I)
}

Received: 1 November 2019 / Revised: 23 January 2020 / Accepted: 10 February 2020 / Published online: 25 February 2020

(C) Springer-Verlag GmbH Germany, part of Springer Nature 2020

U.S. government works are not subject to copyright.

\begin{abstract}
Epinephrine autoinjectors (EAIs) are important first aid medications for treating anaphylaxis. A 10-fold price increase over the past 12 years and evidence that expired EAIs may still contain significant doses of available epinephrine have motivated interest in the efficacy of expired EAIs as treatments of last resort. Degradation of expired EAIs, which can be caused by improper storage conditions, results in various degrees of discoloration of the epinephrine solution. Previous studies have determined that significant epinephrine remains available in expired EAIs, but these have only considered EAIs that show no discoloration. Here, we investigate the potential for colorimetric estimation of available epinephrine dose based on the degree of discoloration in expired EAIs. The correlation of available epinephrine dose and time since expiration date was poor $(r=-0.37)$, as determined by an industry standard UHPLC protocol. Visible absorbance of the samples integrated across the range 430-475 nm correlated well with available epinephrine dose $(r=-0.71)$. This wavelength corresponds to the blue channel of a typical smartphone camera Bayer filter. Smartphone camera images of the EAI solutions in various illumination conditions were analyzed to assign color indices representing the degree of discoloration. Color index of the samples showed similar correlation $(|r|>0.7)$ with available epinephrine dose as that of visible spectrophotometry. Smartphone imaging colorimetry is proposed as a potential point-of-use epinephrine dose estimator for expired and degraded EAIs.
\end{abstract}

Keywords Epinephrine autoinjector (EAI) · Ultra-high-performance liquid chromatography (UHPLC) · Quantitative smartphone imaging $\cdot$ Point-of-care method

\section{Introduction}

Epinephrine is widely used as a first aid drug for the treatment of anaphylaxis $[1,2]$. Anaphylaxis can be triggered by a wide

Electronic supplementary material The online version of this article (https://doi.org/10.1007/s00216-020-02505-y) contains supplementary material, which is available to authorized users.

Christopher A. Baker

chris.baker@utk.edu

1 Department of Chemistry, University of Tennessee, Knoxville, TN 37996, USA

2 Fellow Academy Wilderness Medicine, Wilderness Medical Society; National Park Service, Obed Wild and Scenic River and Big South Fork National River and Recreation Area; Appalachian Mountain Rescue Team, Oak Ridge, TN 37830, USA

3 NeuroNET Research Center, University of Tennessee, Knoxville, TN 37996, USA

4 Department of Biochemistry and Molecular Biology, University of Tennessee, Knoxville, TN 37996, USA variety of stimuli including food, medication, and envenomation [3-7]. Anaphylaxis shows signs of hyperallergic reactions in quick successions and can lead to death $[8,9]$. Epinephrine induces vasoconstriction which helps reduce or prevent upper airway mucosal edema, hypotension, and shock. Epinephrine also has important bronchodilator effects that help relieve laryngospasm, bronchospasm, and cardiac inotropic and chronotropic effects, which also helps in treating hypotension and shock [8]. Fatality in cases of anaphylaxis is correlated with delayed epinephrine administration [9]. Immediate recognition of anaphylaxis and ability to administer epinephrine are vital to saving lives in cases of anaphylaxis, which is why many people prone to severe allergic reaction, as well as first aid providers, carry epinephrine autoinjectors (EAIs). Still, studies suggest that underuse of epinephrine as a first aid drug in cases of anaphylaxis is a significant problem [10].

EAIs typically contain $1 \mathrm{mg} \mathrm{mL} \mathrm{mL}^{-1}$ epinephrine $\left(0.5 \mathrm{mg} \mathrm{mL}^{-1}\right.$ for EpiPen Junior) in saline solution and are marked with expiration dates after which administration of the 
drug is not recommended [11]. Recent price increases of commercial EAIs have triggered interest in the efficacy of expired EAIs. In 2007, average EAI price increased $545 \%$ to $\$ 350$ [12] and by 2016 the price was $\$ 600$ [13]. A study on patient compliance reported that even patients with high awareness of EAI expiration date may still carry expired EAIs and delay replacement $[14,15]$. Several studies have found $50-90 \%$ active ingredient still available in EAIs from 1 to 90 months after their expiration dates [16-18].

Epinephrine oxidizes in the presence of atmospheric oxygen, taking on a pink to brown appearance depending on the extent of oxidation [19]. Similar degradation of EAI contents was observed after prolonged heat exposure [20]. There is evidence to suggest that epinephrine degradation products are well tolerated in the body. Ocular epinephrine drugs can cause pigmentation of the conjunctiva [21,22] and cornea [21-25] due to epinephrine degradation products produced upon light exposure $[26,27]$. The oxidized derivative of epinephrine is melanin-like adrenochrome [23, 28], and the resulting discoloration of the eye is tolerated as a side effect of ocular epinephrine drugs [27]. Although it is advised to not use discolored EAIs [11, 16, 18], the prevalence of expired EAIs due to cost pressures, and the nature of epinephrine as a drug of first aid, presents the possibility that expired and discolored EAIs can be the only option available in emergency and remote settings.

Austere medicine is the practice of medicine in resource-constrained environments, from wilderness areas in North America to third world countries [29]. In austere environments, the ability to procure fresh medicine can be severely hampered, and the conundrum of using whatever medicine is available becomes the issue. Epinephrine is a life-saving drug for patients experiencing anaphylaxis and, if the medicine is discolored or out of date, waiting for replacement epinephrine may not be a viable option. The safety and efficacy of expired and discolored EAIs is not well established, and the current accepted practice is that discolored EAIs should not be administered. Still, austere medical scenarios exist in which discolored medication will be administered, and in such cases the ability to assess the concentration of epinephrine in discolored or expired EAIs using ubiquitous and readily available smartphone technology may save lives.

Simons and coworkers determined the available active ingredient in expired EAIs [18]. 28 EpiPens and 6 EpiPen Juniors were investigated via spectrophotometry and HPLC. Nearly all samples were colorless and free from precipitates, and all contained at least $50 \%$ of the intended epinephrine dose. They concluded that using an expired EAI during an emergency outweighs the potential risk of giving no treatment, presuming no signs of degradation are observed [18, 30]. These studies did not consider extensively expired EAIs that showed substantial degrees of discoloration.
Smartphone imaging is emerging as an approach to colorimetric detection for quantitative analysis. Blood glucose concentration in diabetic patients [31], electrolytes concentration in human sweat [32], and alkaline phosphate concentration in dairy [33] and serum [34] samples have been quantified via smartphone images. Smartphone imaging has also been utilized in applications such as detecting nucleic acids [35], influenza virus counting [36], immunoassay of prostate specific antigens [37], and detection of $\mathrm{Hg}^{2+}$ via smartphone fluorescence microscopy [38-40].

We hypothesized that discolored EAIs present the opportunity for simple colorimetric estimation of the available epinephrine dose by quantitative smartphone imaging, which may facilitate the evaluation of EAIs at the point of care. Here, we investigated expired EAIs showing various degrees of discoloration with the objectives of (1) determining the correlation between available epinephrine and the time elapsed since expiration date, (2) determining the correlation between available epinephrine and the degree of discoloration, and (3) developing a simple colorimetric methodology that utilizes smartphone imaging to estimate available epinephrine dose in discolored EAI solutions.

\section{Experimental section}

\section{Reagents and materials}

Epinephrine, methanol, 1-octanesulfonic acid, $1 \mathrm{~mL}$ polypropylene syringes, and $0.22 \mu \mathrm{m}$ nylon syringe filters were from Fisher Scientific (Suwanee, GA). All aqueous solutions were prepared with 18.2 $\mathrm{M} \Omega$ water from a Milli-Q purification system (Millipore, Bedford, MA). The Luna C18 chromatography column $\left(50 \times 4.6 \mathrm{~mm}, 5 \mu \mathrm{m}\right.$ particle size, $100 \mathrm{~A}^{0}$ pore size) was from Phenomenex Inc. (Torrance, CA).

\section{Expired EAI samples}

Multiple EAIs were obtained from various sources. Expired EAIs were requested, without reference to storage, and it is assumed that most, if not all the EAIs, were carried by patients or EMS in non-temperature controlled environments. After receipt of the EAIs, all syringe units were carefully removed from their delivery unit, placed in sealed Falcon tubes, and stored at room temperature until assayed. Researchers developing the protocols and analyzing the epinephrine samples were blinded to the expiration date until after analysis. These samples represent a real world scenario of EAI storage rather than using EAIs from controlled environments. Time since expiration date for all the EAIs ranged from 12 to 207 months. 


\section{Epinephrine measurements by UHPLC}

The samples were filtered through $0.22 \mu \mathrm{m}$ nylon syringe filters to eliminate any particulates. The filtrates were diluted to $20 \%$ of original concentration with $50 \%$ methanol in water solution. Epinephrine concentrations were determined using an Agilent 1290 infinity series (Agilent Technologies, Inc., Santa Clara, CA) ultra-high-pressure liquid chromatography (UHPLC) instrument equipped with a diode array detector following a US Pharmacopeia (USP) standard protocol [41]. Briefly, isocratic elution was conducted on a C18 column at

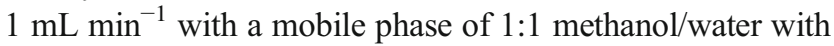
$0.1 \% 1$-octanesulfonic acid added as an ion-pairing agent. UV absorbance was recorded at $278 \mathrm{~nm}$. Epinephrine standard solutions were used to produce a calibration curve in the range from $0.01-1.00 \mathrm{mg} \mathrm{mL}^{-1}$. The concentrations of epinephrine in EAI samples were determined by fitting the resultant peak area to the best fit line of the calibration data.

\section{Characterization by UV-Vis spectrophotometry}

UV-Vis spectra of the EAI samples were obtained using a NanoDrop One spectrophotometer (Thermo Fisher Scientific, Waltham, MA). The absorbance maximum of $278 \mathrm{~nm}$ for epinephrine was determined using epinephrine standard solutions, and a calibration curve was constructed in the concentration range of 0.01 to $1.00 \mathrm{mg} \mathrm{mL}^{-1}$. EAI samples were diluted to $20 \%$ of original concentration to fall within the calibration range. Epinephrine concentrations for expired EAI samples were determined via absorbance at $278 \mathrm{~nm}$ by fitting to the best fit line of the calibration data.

\section{Quantitative smartphone imaging}

Color index was defined as the integrated absorbance of the spectral region $430-475 \mathrm{~nm}$, which corresponds to the blue bandpass component of a common photographic Bayer filter [42]. Smartphone images of the EAI samples were obtained with a Motorola G5S plus device. The Open Camera android application (http://opencamera.org.uk) was used to hold white balance and exposure time constant for all images taken under a particular lighting condition. EAI samples were imaged within standard $1 \mathrm{~mL}$ polypropylene syringes in four different lighting conditions: ambient sunlight, fluorescent office illumination with smartphone flashlight, fluorescent office illumination without smartphone flashlight, and under dark lab conditions while utilizing the smartphone flashlight.

\section{Results and discussion}

Twenty-three expired EAIs, ranging from 12 to 207 months past expiration date, were characterized to determine available epinephrine dose. UHPLC analysis of epinephrine standard solutions via a standardized USP protocol gave a peak at $2.86 \pm 0.01 \mathrm{~min}$ (Fig. 1a). The EAI samples were observed as a range of colored solution from clear to black and were qualitatively categorized into four groups, clear, pink, brown, and black, based on their appearance to the naked eye. Figure 1b-e shows representative chromatograms of samples from each qualitative category. All samples showed a peak at approximately $2.9 \mathrm{~min}$, indicative of available epinephrine in the EAI solution. Increasing color intensity correlated with an increase in additional peaks eluting in the range of 1-2 $\mathrm{min}$. We postulate that these additional peaks are epinephrine degradation by-products, although further study is needed to identify these constituents. EAI samples were evaluated by UHPLC in triplicate to yield concentration determinations with RSD $<5 \%$ in all cases. In this study, epinephrine concentrations in expired EAIs were found to be in the range of $0.05-1.43 \mathrm{mg} \mathrm{mL}^{-1}$. Although it was unexpected to observe epinephrine concentrations higher than the reported dose of $1 \mathrm{mg} \mathrm{mL}^{-1}$, and the nature of this discrepancy is not immediately clear, this finding corroborates those of Simons and coworkers who measured epinephrine concentrations as high as $1.19 \mathrm{mg} \mathrm{mL}^{-1}$ [18]. We found that time since expiration date was a poor predictor of available epinephrine dose in discolored EAIs. Simons and coworkers found a Pearson correlation coefficient $(r)$ of -0.63 indicating moderate correlation between available epinephrine dose and time since expiration date in expired EAIs that had been stored appropriately to prevent sample discoloration and formation of particulates [18]. For discolored EAI solutions, we observed poor correlation $(r=-0.37)$ of available epinephrine dose with time since expiration date (Fig. 1f).

As an initial investigation into the efficacy of photometry for EAI characterizations, epinephrine concentrations were determined by UV-Vis spectrophotometry of the same filtered EAI samples used for UHPLC measurements. UV absorbance spectra are shown in Fig. 2a for the same representative samples shown in Fig. 1b-e, with an additional trace showing an epinephrine standard solution of concentration $0.4 \mathrm{mg} \mathrm{mL}^{-1}$. EAI epinephrine concentrations were determined via calibration of UV absorbance at $278 \mathrm{~nm}$.

Figure $2 b$ shows the comparison of epinephrine concentrations determined by UV spectrophotometry with those determined by the standard UHPLC protocol. UV absorbance consistently overestimated epinephrine concentrations, which is evident from the mostly positive residual values shown in the residual plot of Fig. 2c. We hypothesize that the inaccuracy of UV absorbance in determining epinephrine concentration is a result of spectral overlap between degradation products in solution and epinephrine, which is supported by the observation of non-epinephrine peaks in the chromatograms of degraded EAI samples with absorbance detection at $278 \mathrm{~nm}$ (see Fig. 1b-e). Importantly, UV absorbance was measured 
Fig. 1 Epinephrine quantitation by UHPLC: representative chromatograms of a pure epinephrine standards, b expired EAI sample that appeared black, $\mathbf{c}$ expired EAI sample that appeared brown, $\mathbf{d}$ expired EAI sample that appeared pink, e expired EAI sample that appeared clear, and $\mathbf{f}$ Poor correlation $(r=-0.37)$ was observed for epinephrine concentration in expired and discolored EAI samples vs. time elapsed since expiration date
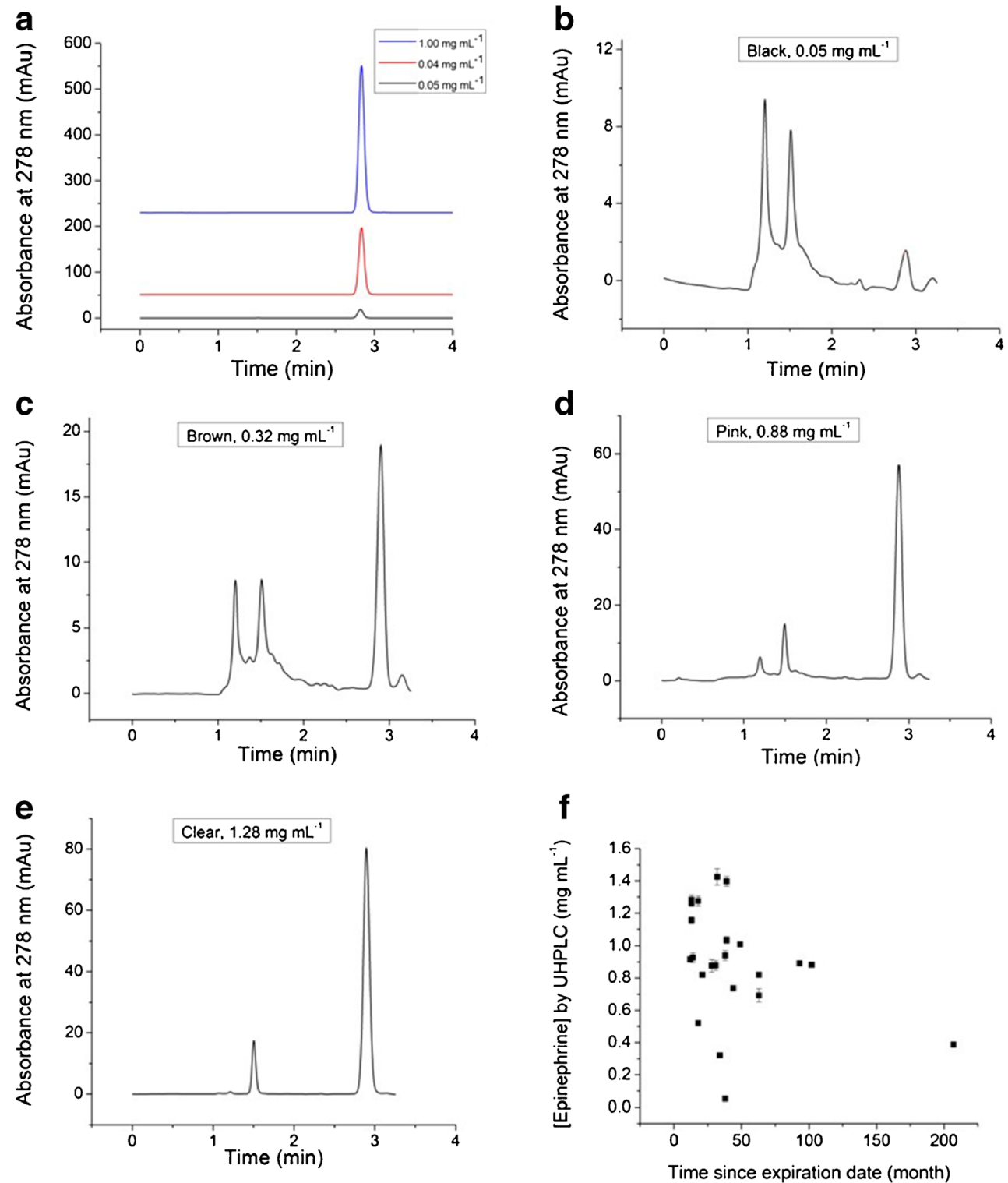

using filtered samples, which eliminated particulate matter and had the effect of reducing, although not eliminating entirely, the intensity of discoloration as observed by the naked eye. Filtration was necessary prior to UHPLC and was convenient for avoiding aberrant scatter in UV absorbance measurements, but it poorly approximated the conditions of expired EAI solutions at the point of use.

We hypothesized that colorimetry of the unfiltered, and thus more intensely colored, expired EAI samples would yield a more effective correlation with epinephrine concentration. Unfiltered samples appeared with various red hues ranging from pink to dark brown; thus, we investigated absorbance in the blue region of the visible spectra. Figure 3 a shows the UV-visible spectra in the range 175$850 \mathrm{~nm}$ and an enlarged view of the range 430-475 nm (inset), which corresponds with the typical bandpass range of the blue component of a Bayer filter found in many digital cameras [43].

The trend of integrated absorbance across the range 430 $475 \mathrm{~nm}$ corresponded with the trend of discoloration intensity, shown in Fig. 3b, and with the trend of epinephrine concentrations as determined by UHPLC (given in Fig. 3a color legend), whereas absorbance at $278 \mathrm{~nm}$ did not show the same correlation with the trend of epinephrine concentrations.

The integrated area of the absorbance spectrum from $430 \mathrm{~nm}$ to $475 \mathrm{~nm}$ was calculated for each sample, and we termed this value the color index. All measured color index values were normalized by dividing by the highest measured color index to give the relative color index, which has a maximum possible value of 1 . Figure 4 shows the correlation between epinephrine concentration as determined by UHPLC and the relative color index value. A significant 
Fig. 2 Epinephrine quantitation by UV spectrophotometry: a representative UV absorbance spectrum of discolored EAI samples and an epinephrine standard solution at $0.4 \mathrm{mg} \mathrm{mL}^{-1}$, b comparison of epinephrine concentrations determined by UV spectrophotometry vs. those determined by the industry standard UHPLC protocol, and c residual plot of epinephrine concentrations of EAI samples from UV spectrophotometry vs. concentrations determined by UHPLC
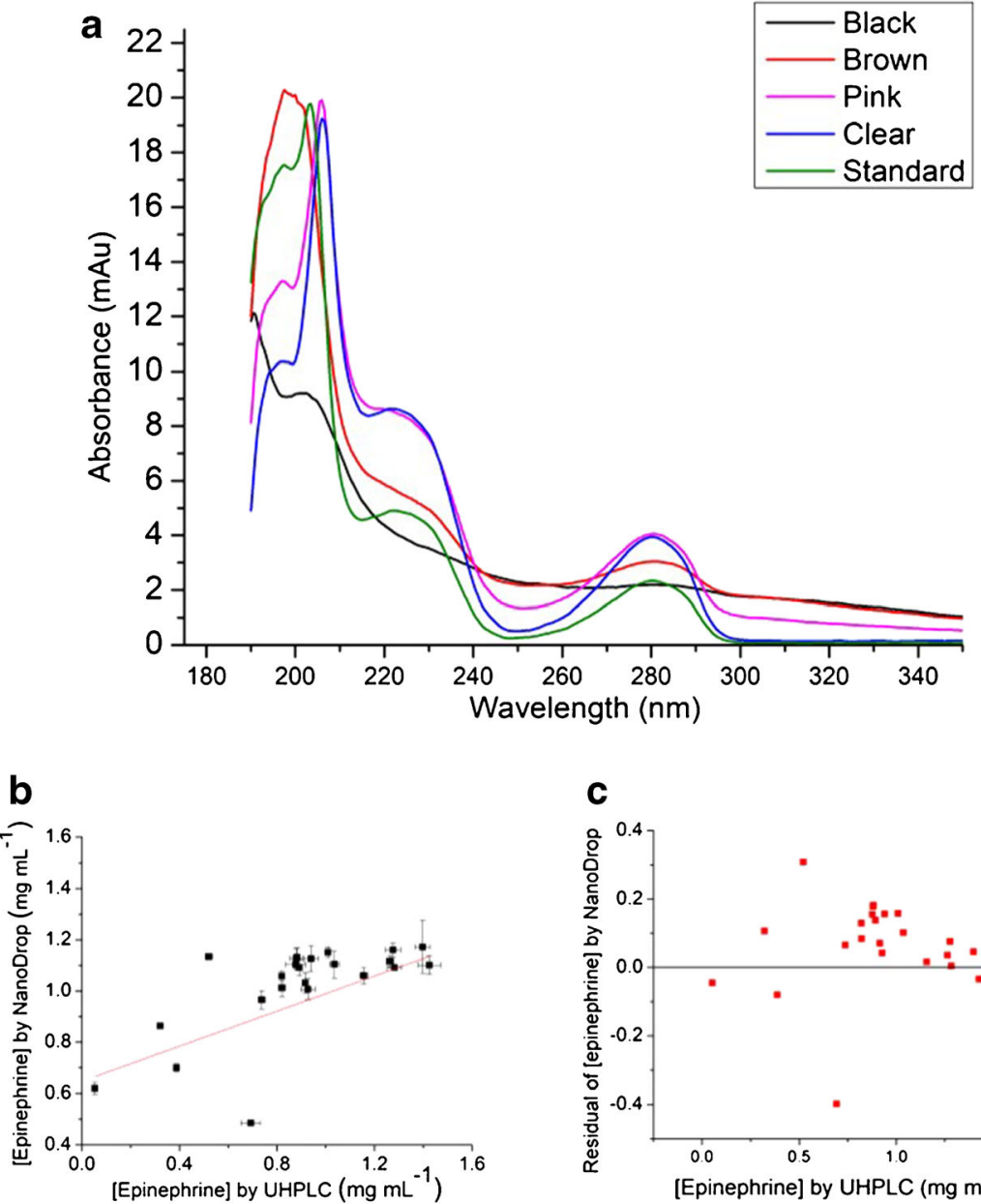

negative correlation is observed as characterized by a Pearson correlation coefficient of -0.71 . This negative correlation suggests that colorimetry of the unfiltered degraded EAI samples may offer an effective approach for estimating epinephrine concentration.

To demonstrate the efficacy of smartphone imaging for colorimetric assay of degraded EAIs, solutions were transferred into standard $1 \mathrm{~mL}$ polypropylene syringes and imaged via smartphone camera. Imaging conditions were standardized by utilizing a sheet of standard A4 printer paper as an imaging background, and multiple illumination conditions were investigated.

Each colored image was decomposed into three grayscale images representing the red, green, and blue components (i.e., converted to "RGB stack") using the open source imaging application Fiji [44]. Mean grayscale intensities from the blue image channel in specific regions of interest were used to determine an approximated color index value as follows:

Color index $=\log _{10} \frac{I_{0}}{I}$ where $I_{0}$ is the mean grayscale intensity from a representative region of interest on the white background, and $I$ is the mean grayscale intensity from a representative area of the syringe as indicated in Fig. 5a-d. This image analysis was performed on images collected outdoors under ambient sunlight (Fig. 5a, e), under fluorescent office illumination with the addition of smartphone flashlight illumination (Fig. 5b, f), under fluorescent office illumination without the addition of smartphone flashlight illumination (Fig. 5c, g), and under dark conditions while utilizing the smartphone flashlight (Fig. 5d, h). As with the data shown in Fig. 3, all calculated color index values were normalized to the highest measured color index to give relative color index. Under all illumination conditions, similar negative correlations are observed between the measured color index and the epinephrine dose as determined via the standard UHPLC protocol, with $r$ ranging from -0.71 to -0.77 . Following the methodology of Watters and coworkers [45], the calibration plots in Fig. 5e-h were each fitted with a $95 \%$ confidence band to estimate confidence interval as a function of epinephrine concentration (see the Electronic Supplementary Material (ESM), Fig. S1). 
Fig. 3 Visible spectrophotometry of discolored EAI solutions: a representative UV-Vis absorbance spectrum of discolored EAI samples, labeled by epinephrine concentration determined via UHPLC. The inset shows a magnified view of the visible spectra in the range $430-475 \mathrm{~nm}$. b Images of samples represented in panel a. Color labels correspond to the line colors of panel a

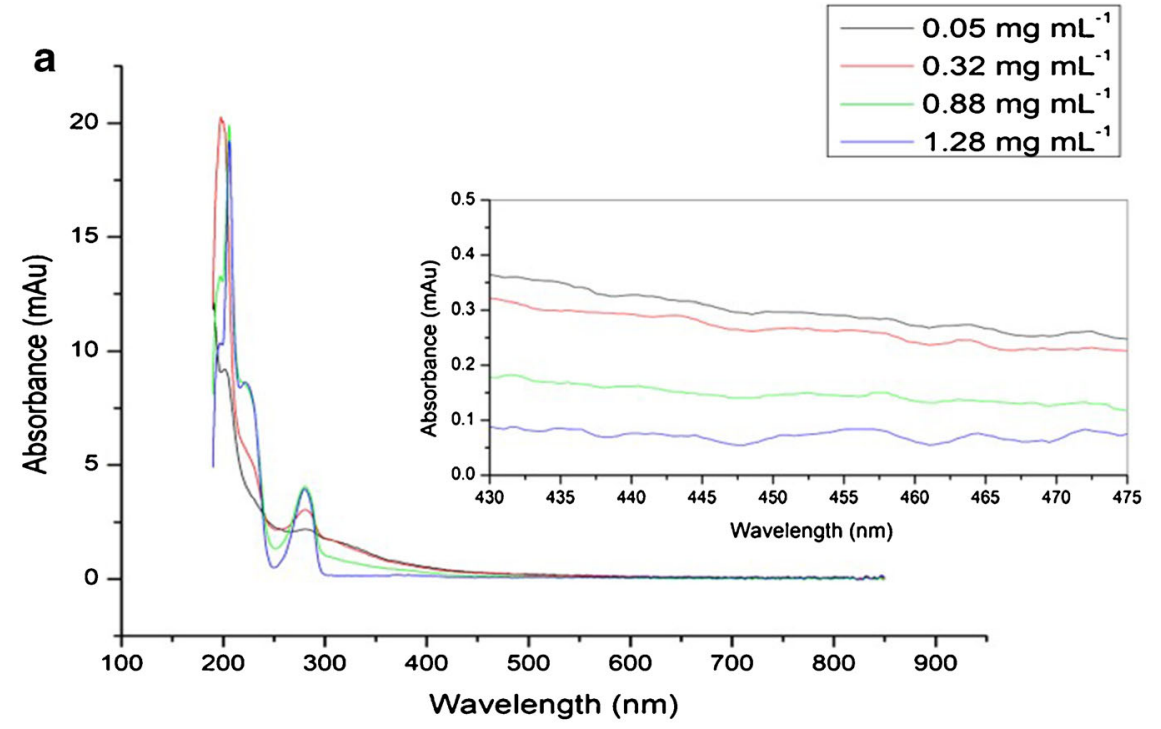

b

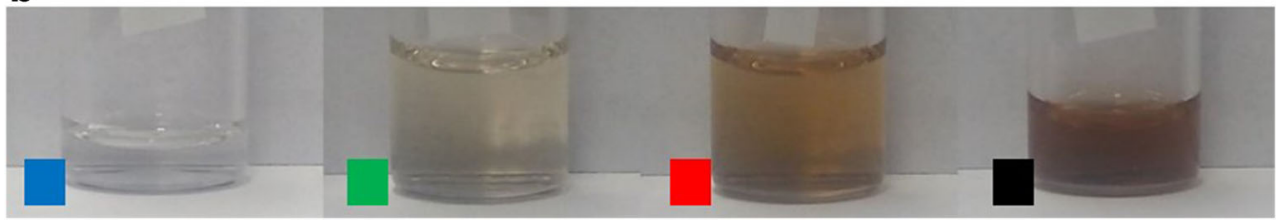

The estimated $95 \%$ confidence interval at $1 \mathrm{mg} \mathrm{mL}^{-1}$ epinephrine ranged from \pm 0.12 to $\pm 0.15 \mathrm{mg} \mathrm{mL}^{-1}$ for all lighting conditions studied here, indicating that, dependent on lighting conditions, a $12-15 \%$ reduction in reported epinephrine dose can be detected with $95 \%$ confidence. There are two important points to note here. First, these calibrations are intended to demonstrate proof of principle, and further calibration from a wider variety of sample

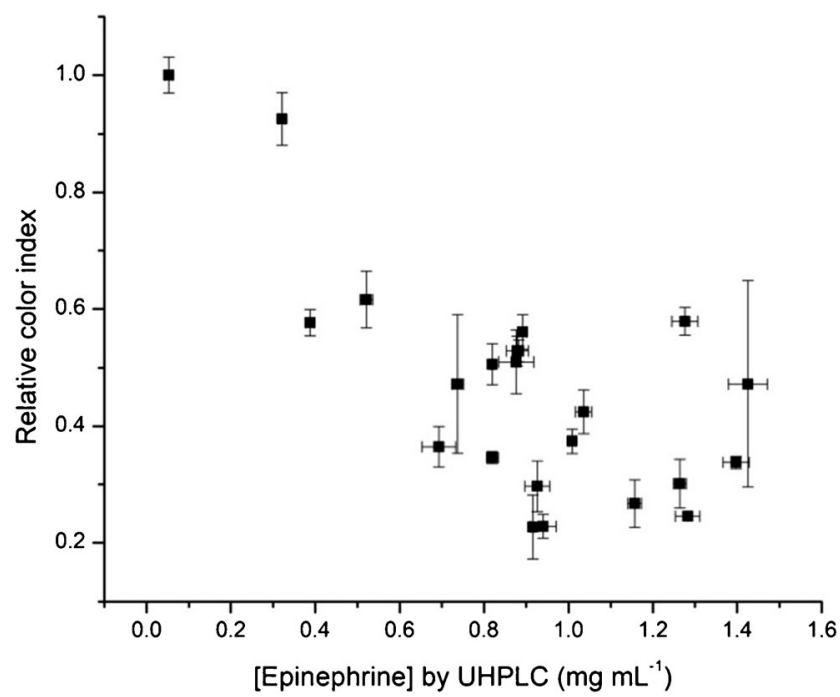

Fig. 4 Relative color index vs. epinephrine concentration: color index correlates well with epinephrine concentration as determined by UHPLC, giving a Pearson correlation coefficient of $r=-0.71$ sources is needed to deliver an assay with clinical utility. Second, there are no empirical limits established on effective epinephrine dose because epinephrine dosing for the treatment of anaphylaxis is not based on empirical study of the dose-response relationship [46]. Therefore, no empirical metric exists by which to determine whether the demonstrated assay performance is sufficient for clinical utility. Still, in-date EAIs have been reported to contain in the range of $86-114 \%$ of the stated epinephrine dose [18] indicating that the current assay can determine epinephrine dose degradation to within the accuracy of dosage of unexpired EAIs.

A quantitative smartphone imaging assay can enable colorimetric estimation of epinephrine in environments where imaging conditions could be difficult to control. Thus, we investigated the robustness of this assay to sample position and orientation with respect to the smartphone camera (Fig. 6). Four representative samples of various hue (i.e., clear, pink, brown, and black in appearance) were studied to determine the variation of color index as a function of the syringe distance from the smartphone camera. We estimated that a natural ergonomic range of camera-sample distance for handheld image acquisition was $15-45 \mathrm{~cm}$, and images were recorded with the syringe placed at $7.5 \mathrm{~cm}$ increments within that range. For all images, the white background was placed $3.8 \mathrm{~cm}$ behind the syringe, which we estimated as an appropriate distance to represent a syringe and white paper background held in the same hand approximately one-to-two 
a
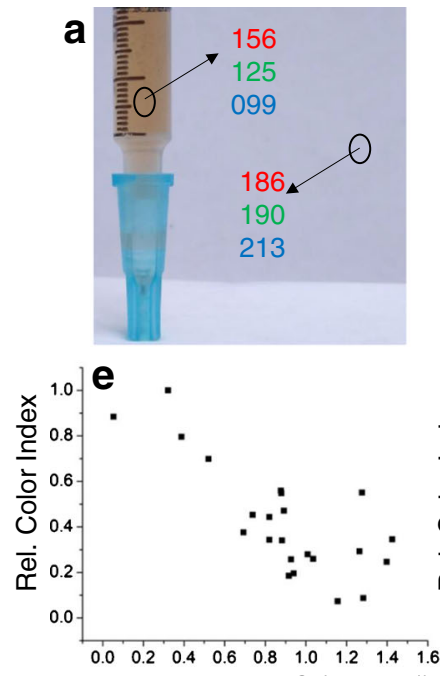

[Epinephrine] by UHPLC $\left(\mathrm{mg} \mathrm{mL}^{-1}\right)$ b
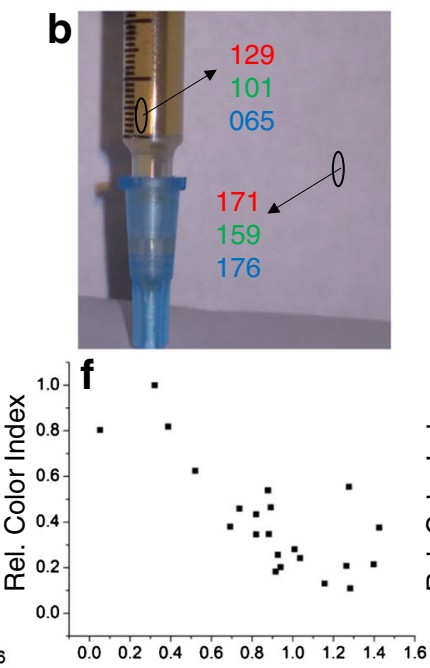

[Epinephrine] by UHPLC $\left(\mathrm{mg} \mathrm{mL}^{-1}\right)$

Fig. 5 Quantitative smartphone imaging of discolored EAI solutions: Representative images of EAI solutions illuminated via a ambient sunlight, b fluorescent office illumination with smartphone flashlight, $\mathbf{c}$ fluorescent office illumination without smartphone flashlight, and $\mathbf{d}$ dark ambient conditions with smartphone flashlight illumination. Plots of relative color index vs. epinephrine concentration under e ambient sunlight $(r=-0.77)$, f fluorescent office illumination with smartphone

finger widths apart. Figure 6a shows small but statistically significant differences in color index for the clear sample only when comparing images recorded at $22.5 \mathrm{~cm}$ to those recorded at $37.5 \mathrm{~cm}(p=0.007)$. Statistically significant differences were observed for the brown sample when comparing images recorded at $30 \mathrm{~cm}$ to either those recorded at $15 \mathrm{~cm}(p=0.002)$ or those recorded at $45 \mathrm{~cm}(p=0.01)$, but no other significant differences were observed in the study of camera-sample distance. When color index values from all sample distances

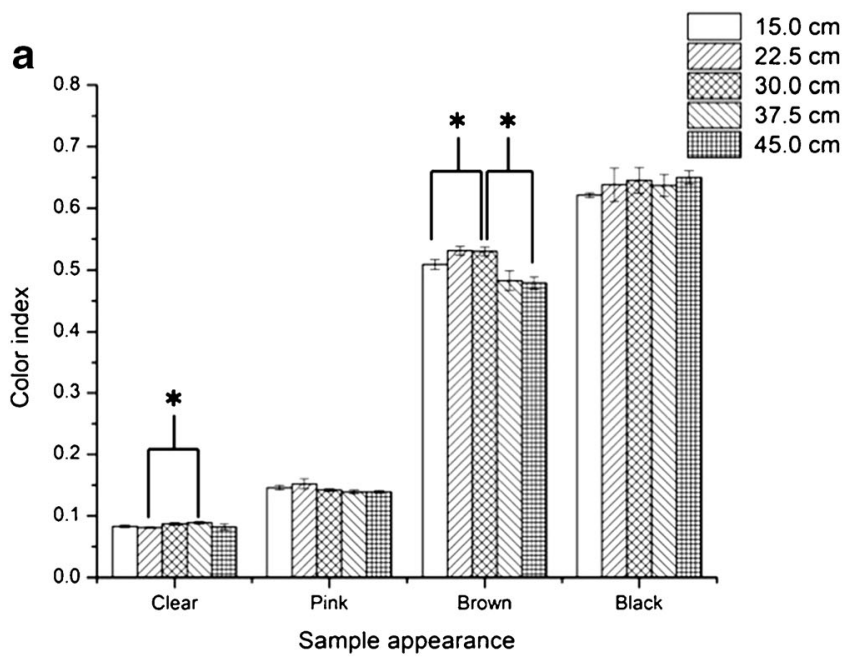

Fig. 6 Influence of sample distance and orientation on color index determinations: color index values were determined for representative samples shown in Fig. 3 while varying a imaging distance and $\mathbf{b}$ syringe orientation with respect to the camera. All bars within each

C
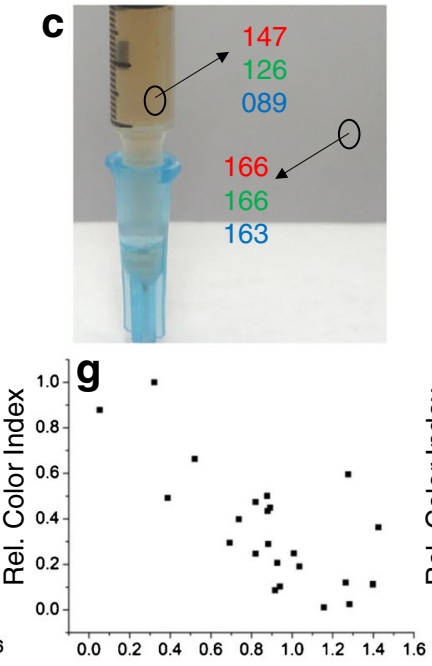

d
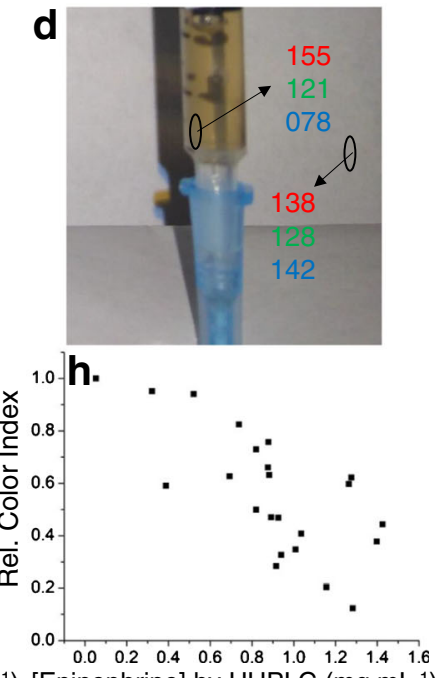

[Epinephrine] by UHPLC (mg mL-1) [Epinephrine] by UHPLC $\left(\mathrm{mg} \mathrm{mL}^{-1}\right)$

flashlights $(r=-0.76)$, $g$ fluorescent office illumination without smartphone flashlights $(r=-0.71)$, and $\mathbf{h}$ dark ambient conditions with smartphone flashlight illumination $(r=-0.71)$. Black circles in a-d indicate regions of interest that gave mean intensity values for $\mathrm{R}, \mathrm{G}$, and $\mathrm{B}$ as indicated in each figure. Note smaller regions of interest were used in panels $\mathbf{b}$ and $\mathbf{d}$ so as to avoid the effects of glare on the syringe surface

were pooled for each sample color, RSD was $4.8 \%, 4.3 \%$, $4.9 \%$, and $2.9 \%$ for clear, pink, brown, and black samples, respectively. This result suggests that, despite the small significant differences observed, the imaging assay is robust to camera-sample distance in the range of 15-45 cm.

We estimated that an operator could reasonably approximate syringe orientation to within $\pm 20^{\circ}$ of vertical. Thus, to study the influence of syringe orientation, we collected sample images with syringes oriented vertically and with $20^{\circ}$ of tilt in

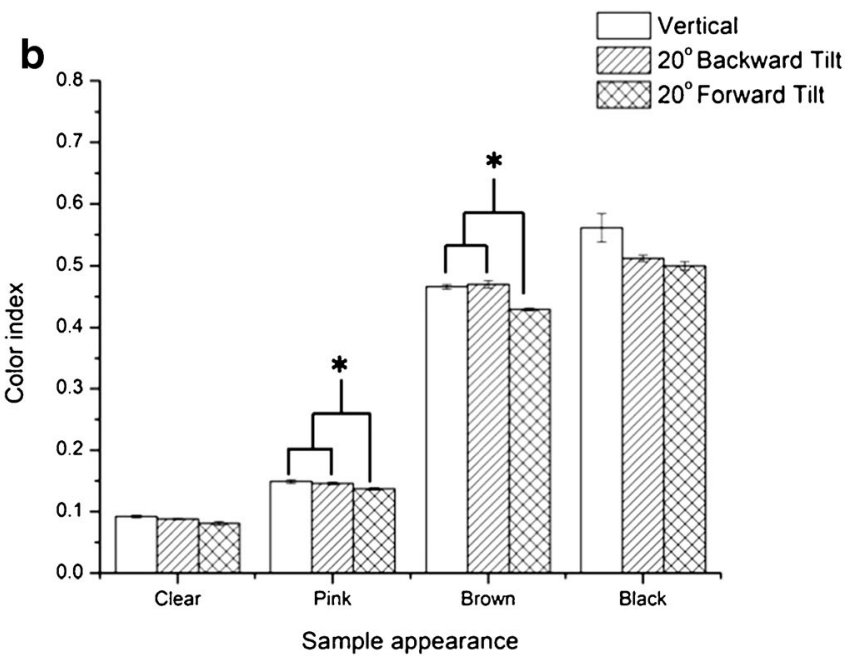

sample color were compared via paired two sample two-tailed $t$ test, and statistically significant differences $(p<0.05)$ were observed between the bars indicated with an asterisk $(*)$ 
the plane of the image (i.e., left and right tilt) and out of the plane of the image (i.e., forward and back tilt). There were no statistically significant differences observed when comparing data from vertically oriented syringes with those tilted left or right, within the plane of the image (data not shown). This is unsurprising, since tilted orientation within the plane of the image does not affect the path length of light from the white background through the volume of the syringe. Statistically significant reductions in color index value $(p<0.005)$ were observed when comparing syringes tilted forward (i.e., toward the camera) with either vertical or backward tilted syringe images for the pink and brown samples only (Fig. 6b). The origin of this phenomenon is not immediately clear, but we do not attribute it to increased path length which would increase the observed color index value. When color index values from all sample orientations were pooled for each sample color, RSD was $5.0 \%, 3.4 \%, 4.3 \%$, and $7.5 \%$ for clear, pink, brown, and black samples, respectively. Thus, the assay is robust to sample orientation when the syringe is oriented within $\pm 20^{\circ}$ of vertical.

\section{Conclusions}

We investigated available epinephrine dose in expired and discolored EAIs via the USP standard UHPLC methodology, spectrophotometry, and quantitative smartphone imaging. We found that time past expiration date is a poor predictor of available epinephrine concentration when EAIs have degraded and become discolored. Although spectrophotometry is employed for quality control in commercial epinephrine production [47], UV absorbance at $278 \mathrm{~nm}$ did not accurately estimate the epinephrine concentration in degraded EAIs, likely due to the presence of degradation products with spectral overlap with epinephrine. In discolored and unfiltered EAIs, visible absorbance in the range $430-475 \mathrm{~nm}$ correlated well with available epinephrine concentration, which enabled the development of a quantitative smartphone imaging protocol. A simple digital image processing procedure utilizing smartphone images yielded a strong correlation $(|r|>0.7)$ with epinephrine concentrations determined by standard UHPLC protocols, and this strong correlation persisted under various lighting conditions. Quantitative smartphone imaging was robust to moderate variations in sample-camera distance and sample orientation, yielding color index determination with RSD $\leq 7.5 \%$ in all cases studied.

The present work stops short of providing a point-of-use analysis tool for several important reasons. First, although our work demonstrates feasibility for quantitative imaging in various lighting conditions, a universal calibration that is normalized for illumination and white balance conditions is still needed. This presents a multivariate challenge that will form the basis of future work in this area. Second, although the digital image processing employed here is well within the computational abilities of common smartphones, in the present work digital image processing was accomplished offline using a personal computer. Substantial development is needed to package the image processing tasks along with an appropriate user interface into a simple-to-use smartphone application. Again, we intend to address this challenge with future work. Finally, and most importantly, the safety and efficacy of administering discolored EAI drugs is not well established. Although we postulate that the tolerance of discolored degradation products in ocular epinephrine drugs suggests low toxicity of the degradation products, this determination requires consideration by the wider medical community and lies well beyond the scope of the present work. Still, the present work establishes two key findings: (1) EAI discoloration can be utilized as an indicator for approximating available epinephrine concentration and (2) EAI discoloration is readily quantified via a simple and robust smartphone imaging procedure.

Acknowledgments We would like to thank Dr. Seth C. Hawkins MD for his interest in the project and supplying the EAIs that were used in testing.

Funding information This work was supported by start-up funding provided by the University of Tennessee, College of Arts and Sciences, and the Department of Chemistry.

\section{Compliance with ethical standards}

Conflict of interest The authors declare that they have no conflicts of interest.

\section{References}

1. Simons FE. Epinephrine (adrenaline) in the first-aid, out-of-hospital treatment of anaphylaxis. Novartis Found Symp. 2004;257:228-43 discussion 43-7, 76-85.

2. Lieberman A, Marks A, Peet A. Mark's basic medical biochemistry: a clinical approach. 4th ed. Philadelphia: Wolters Kluwer Health/Lippincott Williams \& Wilkins; 2013.

3. Boden SR, Wesley Burks A. Anaphylaxis: a history with emphasis on food allergy. Immunol Rev. 2011;242(1):247-57.

4. Golden DB, Marsh DG, Kagey-Sobotka A, Freidhoff L, Szklo M, Valentine MD, et al. Epidemiology of insect venom sensitivity. JAMA. 1989;262(2):240-4.

5. Barnard JH. Studies of 400 Hymenoptera sting deaths in the United States. J Allergy Clin Immunol. 1973;52(5):259-64.

6. Sampson HA, Munoz-Furlong A, Bock SA, Schmitt C, Bass R, Chowdhury BA, et al. Symposium on the definition and management of anaphylaxis: summary report. J Allergy Clin Immunol. 2005;115(3):584-91.

7. Sampson HA. Anaphylaxis and emergency treatment. Pediatrics. 2003;111(6 Pt 3):1601-8.

8. Sicherer SH, Simons FER. Epinephrine for first-aid management of anaphylaxis. Pediatrics. 2017;139(3).

9. Lieberman PL. Recognition and first-line treatment of anaphylaxis. Am J Med. 2014;127:S6-11. 
10. Prince BT, Mikhail I, Stukus DR. Underuse of epinephrine for the treatment of anaphylaxis: missed opportunities. J Asthma Allergy. 2018;11:143-51.

11. What is epinephrine? Mylan. 2019. https://www.epipen.com/en/ about-epipen-and-generic/what-is-epinephrine. Accessed June 2019.

12. Pepper AN, Westermann-Clark E, Lockey RF. The high cost of epinephrine autoinjectors and possible alternatives. J Allergy Clin Immunol Pract. 2017;5(3):665.

13. Rubin R. EpiPen price hike comes under scrutiny. Lancet. 2016;388(10051):1266.

14. Noone SA, Sicherer SH. Patient compliance with self-administered epinephrine (SAE) for food allergic (FA) children. J Allergy Clin Immunol. 1999;103(1):S54.

15. Huang SW. A survey of Epi-PEN use in patients with a history of anaphylaxis. J Allergy Clin Immunol. 1998;102(3):525-6.

16. Patadia DD, Stukus DR. Are expired EpiPens still viable? J Allergy Clin Immunol Pract. 2017;5(5):1469-70.

17. Rachid O, Simons FER, Wein MB, Rawas-Qalaji M, Simons KJ. Epinephrine doses contained in outdated epinephrine auto-injectors collected in a Florida allergy practice. Ann Allergy Asthma Immunol. 2015;114(4):354.

18. Simons FER, Gu XC, Simons KJ. Outdated EpiPen and EpiPen Jr autoinjectors: past their prime? J Allergy Clin Immunol. 2000;105(5):1025-30.

19. American Society of Health System Pharmacists. Bethesda, MD: AHFS Drug Information; 2009.

20. Parish HG, Bowser CS, Morton JR, Brown JC. A systematic review of epinephrine degradation with exposure to excessive heat or cold. Ann Allergy Asthma Immunol. 2016;117(1):79-87.

21. Corwin ME, Spencer WH. Conjunctival melanin depositions - a side-effect of topical epinephrine therapy. AMA Arch Ophthalmol. 1963;69(3):317.

22. Veirs ER, McGrew JC. Ocular complications from topical epinephrine therapy of glaucoma. Eye Ear Nose Throat Mon. 1963;42(9): 46-52.

23. Ferry AP, Zimmerman LE. Black cornea - complication of topical use of epinephrine. Am J Ophthalmol. 1964;58(2):205.

24. Reinecke RD. Kuwabara T. Corneal deposits secondary to topical epinephrine. AMA Arch Ophthalmol. 1963;70(2):170.

25. Cleasby G, Donaldson DD. Epinephrine pigmentation of the cornea. JAMA Ophthalmol. 1967;78(1):74-5.

26. Bullock JD. Epinephrine pigmentation. JAMA Ophthalmol. 1970;84(4):546.

27. Bernstein HN. Epinephrine pigmentation-reply. AMA Arch Ophthalmol. 1970;84(4):546.

28. Garnayak S, Patel S. Oxidation of epinephrine to adrenochrome by cetyltrimethylammonium dichromate: a mechanistic study. Ind Eng Chem Res. 2014;53(31):12249-56.

29. https://www.hopkinsmedicine.org/som/wildernessmedicine/index. html. Accessed 7 Oct 2019.

30. Pietroski N. Expired drugs: immortal or DOA? Wilderness Medicine Magazine. 2015 November 29.

31. Wang TT, Lio CK, Huang H, Wang RY, Zhou H, Luo P, et al. A feasible image-based colorimetric assay using a smartphone RGB camera for point-of-care monitoring of diabetes. Talanta. 2020;206.

32. Sekine Y, Kim SB, Zhang Y, Bandodkar AJ, Xu S, Choi J, et al. A fluorometric skin-interfaced microfluidic device and smartphone imaging module for in situ quantitative analysis of sweat chemistry. Lab Chip. 2018;18(15):2178-86.

33. Mahato K, Chandra P. Paper-based miniaturized immunosensor for naked eye ALP detection based on digital image colorimetry integrated with smartphone. Biosens Bioelectron. 2019;128:9-16.

34. Hou L, Qin YX, Li JY, Qin SY, Huang YL, Lin TR, et al. A ratiometric multicolor fluorescence biosensor for visual detection of alkaline phosphatase activity via a smartphone. Biosens Bioelectron. 2019;143.

35. Priye A, Ball CS, Meagher RJ. Colorimetric-luminance readout for quantitative analysis of fluorescence signals with a smartphone CMOS sensor. Anal Chem. 2018;90(21):12385-9.

36. Minagawa $\mathrm{Y}$, Ueno $\mathrm{H}$, Tabata KV, Noji H. Mobile imaging platform for digital influenza virus counting. Lab Chip. 2019;19(16): 2678-87.

37. Lv SZ, Zhang KY, Tang DP. A new visual immunoassay for prostate-specific antigen using near-infrared excited CuxS nanocrystals and imaging on a smartphone. Analyst. 2019;144(12):3716-20.

38. Shan YK, Wang B, Huang HC, Jiang D, Wu XP, Xue L, et al. Onsite quantitative $\mathrm{Hg} 2+$ measurements based on selective and sensitive fluorescence biosensor and miniaturized smartphone fluorescence microscope. Biosens Bioelectron. 2019;132:238-47.

39. Ravindranath R, Periasamy AP, Roy P, Chen YW, Chang HT. Smart app-based on-field colorimetric quantification of mercury via analyte-induced enhancement of the photocatalytic activity of TiO2-Au nanospheres. Anal Bioanal. 2018;410(18):4555-64.

40. Lee WI, Park Y, Park J, Shrivastava S, Son YM, Choi HJ, et al. A smartphone fluorescence imaging-based mobile biosensing system integrated with a passive fluidic control cartridge for minimal user intervention and high accuracy. Lab Chip. 2019;19(8):1502-11.

41. Convention USP. USP35 NF30, 2012: U. S. Pharmacopoeia National Formulary. United States Pharmacopeial; 2011.

42. Xiaoqian W, Fan F, Jiatong S, Lu W, Srivastava A, Chigrinov VG. Evaluation of LC Fresnel phase plate utilized as colour filter. 2012.

43. Cheremkhin PA, Lesnichii VV, Petrov NV. Use of spectral characteristics of DSLR cameras with Bayer filter sensors. J Phys Conf Ser. 2014;536:012021.

44. Schindelin J, Arganda-Carreras I, Frise E, Kaynig V, Longair M, Pietzsch T, et al. Fiji: an open-source platform for biological-image analysis. Nat Methods. 2012;9:676.

45. Watters RL, Carroll RJ, Spiegelman CH. Error modeling and confidence interval estimation for inductively coupled plasma calibration curves. Anal Chem. 1987;59(13):1639-43.

46. Lieberman P, Nicklas RA, Randolph C, Oppenheimer J, Bernstein $\mathrm{D}$, Bernstein $\mathrm{J}$, et al. Anaphylaxis - a practice parameter update 2015. Ann Allergy Asthma Immunol. 2015;115(5):341-84.

47. The United States Pharmacopeia: USP 24: the National Formulary: NF 19 : by authority of the United States Pharmacopoeial Convention, Inc., meeting at Washington, D.C., March 9-12, 1995 ; prepared by the Committee of Revision and published by the Board of Trustees. Rockville, Md.: United States Pharmacopeial Convention; 1999.

Publisher's note Springer Nature remains neutral with regard to jurisdictional claims in published maps and institutional affiliations. 